\title{
Analysis of the Optical Quartz Lens Centering Process Based on Acoustic Emission Signal Processing and the Support Vector Machine
}

Chun-Wei Liu

National Tsing-Hua University: National Tsing Hua University

Shiau-Cheng Shiu (D s109033802@m109.nthu.edu.tw)

National Tsing-Hua University: National Tsing Hua University

Kai-Hung Yu

National Tsing-Hua University: National Tsing Hua University

\section{Research Article}

Keywords: Centring, Biplane quartz lens, Grinding wheel wear, AE signal, Material removal, SVM

Posted Date: July 21st, 2021

DOl: https://doi.org/10.21203/rs.3.rs-719030/v1

License: (c) (i) This work is licensed under a Creative Commons Attribution 4.0 International License.

Read Full License

Version of Record: A version of this preprint was published at The International Journal of Advanced Manufacturing Technology on January 20th, 2022. See the published version at https://doi.org/10.1007/s00170-021-08385-5. 


\title{
Analysis of the Optical Quartz Lens Centering Process Based on Acoustic Emission Signal Processing and the Support Vector Machine
}

\author{
Chun-Wei Liu ${ }^{1, \mathrm{a}}{ }^{*}$, Shiau-Cheng Shiu ${ }^{1, \mathrm{~b}}$, Kai-Hung Yu ${ }^{1, \mathrm{c}}$ \\ ${ }^{1}$ National Tsing Hua University, Power Mechanical Engineering, Section 2, Guangfu Road, Hsinchu 300044, \\ Taiwan

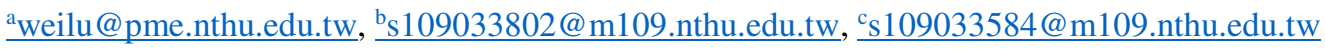

\begin{abstract}
A method was proposed for analyzing the optical glass lens centering process, and experiments on biplane quartz lenses were performed to determine the material removal rate (MRR) for the hard, brittle material. This study used acoustic emission-sensing technology to monitor the MRR and reconstruct the original shape of the lens. The MRR was evaluated, and an error of $17.87 \%$ was obtained. A Taguchi experiment was combined with signal analysis to optimize the process parameters, and a support-vector machine was trained to classify the quality of the grinding wheel; the model had accuracy $98.8 \%$. By using the proposed analysis method, workpiece quality was controlled to an edge surface roughness of $<2 \mu \mathrm{m}$, a lens circularity error of $<0.01 \mathrm{~mm}$, a crack length of $<\mathrm{E} 0.1$, and an optical axis error of $<150 \mu \mathrm{rad}$.
\end{abstract}

Keywords: Centring, Biplane quartz lens, Grinding wheel wear, AE signal, Material removal, SVM

\section{Introduction}

Biplane quartz lens is an important auxiliary-material in semiconductor manufacturing processes. With high purity (impurity < 10ppm), low thermal expansion coefficient $\left(5.11 \sim 5.8^{*} 10^{-7} / \mathrm{K}\right)$, high temperature resistance (softening point about $1700^{\circ} \mathrm{C}$ ), high resistance to acids and alkalis, low refractive index $(1.45)$, low dispersion (Abbe number $\left.=68\right)$ and good light transmittance in the infrared to ultraviolet bands, quartz is often used in experimental equipment and highprecision measuring instruments. Among the manufacturing processes of biplane quartz lens, centring, which minimizes optical axis error, is the key procedure in optical glass lens manufacturing process.

Biplane quartz lenses are a crucial auxiliary material in semiconductor manufacturing processes. Because it has high purity (impurity $<10 \mathrm{ppm}$ ), a low thermal expansion coefficient $\left(5.11-5.8 \times 10^{-7} / \mathrm{K}\right)$, high temperature resistance (softening point of approximately $1,700{ }^{\circ} \mathrm{C}$ ), high resistance to acids and alkalis, a low refractive index (1.45), low dispersion (Abbe number $=68$ ), and good light transmittance in the infrared to ultraviolet bands, quartz is often used in experimental equipment and high-precision measuring instruments. In biplane quartz lens manufacturing processes, centering, which minimizes the optical axis error, is a key procedure.

The complex grinding mechanism and interference from cutting fluids make the centering process difficult to monitor. Because of a lack of real-time monitoring methods, qualities such as the optical axis and the presence of cracks and scratches cannot be observed until the centering process is complete. To solve this problem, acoustic emission (AE) sensors can be applied to monitor the machining process. In the grinding process, AE signals are employed to monitor material removal [1-3], grinding wheel wear [4], chip loading [5-7], and the dressing of the grinding wheel [8]. The root mean square (RMS) of the amplitude of the AE signal is a useful reference value for analyzing time-domain features [9]. 
The surface of a processed material can be evaluated using an AE polar map in ultraprecision turning [10]. The frequency characteristics of AE signals are obtained through fast Fourier transform (FFT) analysis [11, 12]. Wavelet analysis is a suitable method for locally analyzing both the frequency and time domains [13-16]. The time and frequency features of signals can be used as reference parameters of a support vector machine (SVM) to perform binary classification that helps judging certain conditions, such as end point detection [17], tool breakage [18], grinding burns [19-21] and surface roughness monitoring. [22-24]. In complicated situations, SVMs are used to classify multiple classes through a series of steps [25].

This study created an analysis method for the centering process after conducting a series of experiments. AE sensors were used to monitor the amount of lens material removed by the grinding wheel. By accumulating AE signals in the angular domain, the amount of material removed from the lens could be determined, and the original shape could then be inversely calculated. A Taguchi experiment was designed in which the process parameters were used as the input factors for evaluating the influence of each factor on lens quality. The ideal process conditions were determined in advance on the basis of the results of the experiment. A wavelet analysis was performed to decompose the AE signals into seven detailed layers, which indicated the distribution of the signal frequency bands, and the energy ratio of each layer was extracted. An SVM model was created to evaluate the wear caused by the grinding wheel by classifying grinding wheel condition into "normal" or "worn."

\section{Experimental Methods}

\subsection{Grinding Material Removal Rate}

The cylindrical grinding mechanism in the centering process consists of two stages: the infeed and spark-out. Fig. 1 presents a schematic of the cylindrical grinding mechanism. During the infeed stage, the grinding wheel is implemented until the desired outer diameter of the lens is achieved. The spark-out procedure ensures the roundness of lens. The grinding wheel stops infeeding, and material is removed through rotation of the lens and grinding wheel.

The material removal rate (MRR) $u$ is expressed as follows: [26]

$$
u=b_{w} \times a_{e} \times v_{w}
$$

where $b_{w}$ is the grinding width, $a_{e}$ is the effective grinding depth, and $v_{w}$ is the horizontal speed of the workpiece.

In general, the feed rate and rotational speed of the workpiece are fixed during one stage of grinding. If the grinding wheel and workpiece remain in contact, the cutting depth should be the same as the grinding wheel infeed, which stably increases as a function of time. When grinding wheel wear is ignored, $a_{e}$ is expressed as follows:

$$
a_{e}=v_{f} \times t
$$

where $v_{f}$ is the infeed rate of the grinding wheel.

If the lens is rotated several times in one stage, the cutting depth of previous rotations should be subtracted from the total infeed as follows:

$$
a_{e}=v_{f} \times\left(t-\frac{k-1}{n_{w}}\right),
$$

where $n_{w}$ is the rotational speed of the workpiece in rpm, and $k$ is the number of rotations. 


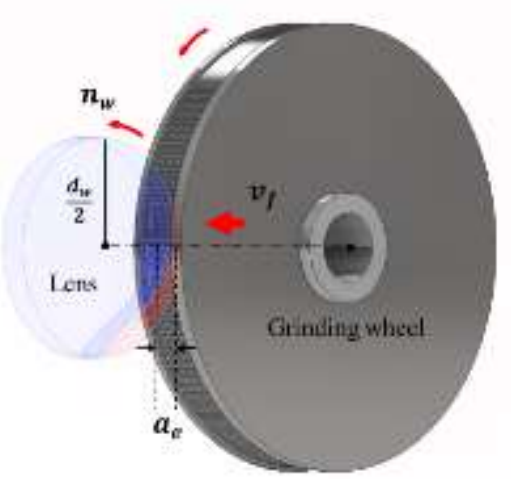

Fig 1. Cylindrical grinding.

A double-plane mirror was manufactured from a square quartz block. Centering is the key process in trimming the outer edge to a circular shape. The diameter of the biplane quartz lens after grinding was $37.65 \mathrm{~mm}$. Fig. 2 presents the biplane quartz lens.

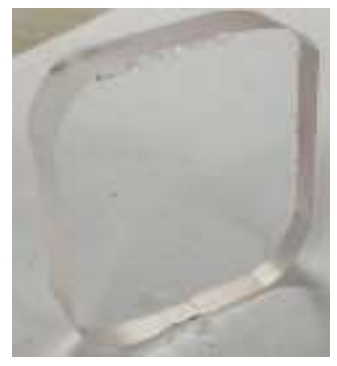

(a)

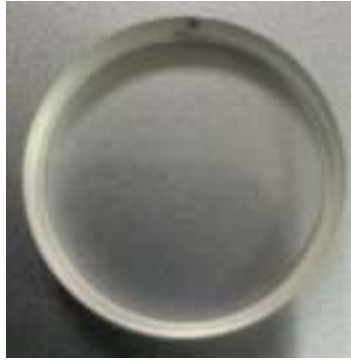

(b)

Fig 2. Biplane quartz lens (a) before and (b) after grinding.

The material removal process is divided into two states based on the geometry of the lens and the grinding process: intermittent removal and continuous removal. Three circles were marked on the polished surface of the lens (Fig. 3):

(1) Holding circle: the diameter of the circle is the holder diameter. The holding force directly affects the number of scratches on the polished surface of the lens.

(2) Desired diameter: the final diameter of the lens set by the working parameters.

(3) Cylindrical grinding circle: the material removal process changes from intermittent removal to continuous removal. Cylindrical grinding starts as the grinding wheel reaches the cylindrical grinding circle. 


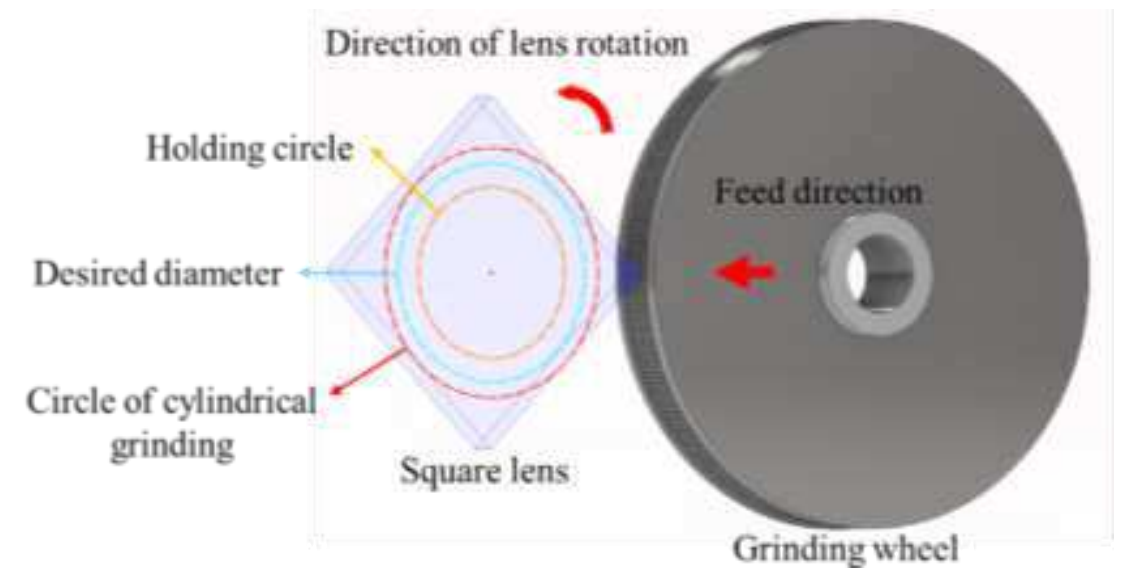

Fig 3. Grinding of square lens

The effective cutting depth varies each time because the contact between the lens and grinding wheel is discontinuous. Because the timing of the cut-in and cut-out at each corner is difficult to determine, AE sensors are used to monitor the contact between the lens and grinding wheel.

The Fig 4 present the details of the lens removal process. Because the lens is rotated about the work axis, the contour of the lens during grinding consists of several concentric circles continuously shrinking inward. The grinding wheel is assumed to make contact with the lens at corner 1 . When corner 2 is rotated to the far right, the grinding wheel covers a horizontal distance $\frac{15 f}{\omega}(\mathrm{mm})$, where $f$ is the infeed rate in $\mathrm{mm} / \mathrm{s}$ and $\omega$ is the lens rotation speed in rpm. When corner 3 is rotated to the far right, the grinding wheel covers $\frac{30 f}{\omega}(\mathrm{mm})$, and so on. The $n$th time a certain corner of the lens is ground, the total feed of the grinding wheel is $\frac{15 n f}{\omega}(\mathrm{mm})$. An arrow-shaped area of the corner is removed, and the central length of the arrow-shaped area is the distance that the grinding wheel has traveled.

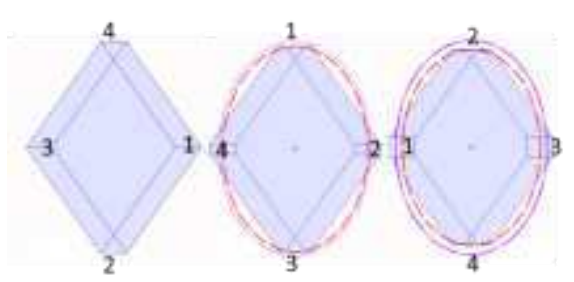

(a) (b) $\quad$ (c)

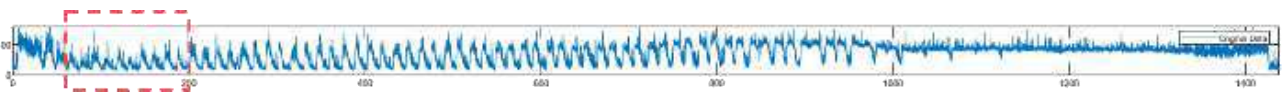

(d)

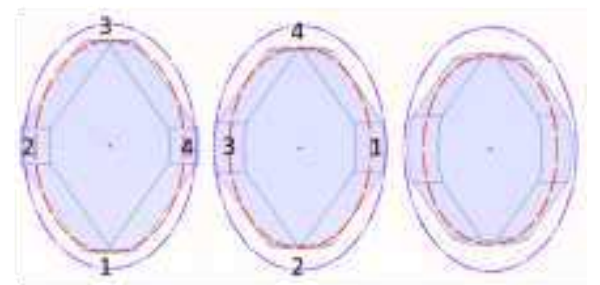

(e) (f)

(g) 


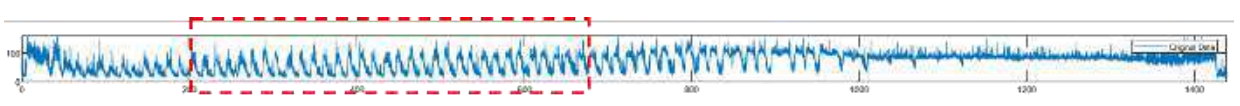

(h)

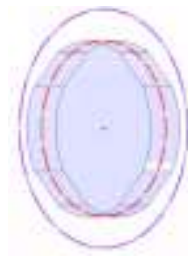

(i)

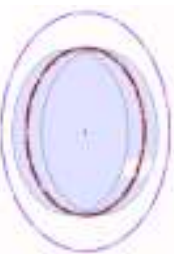

(j)

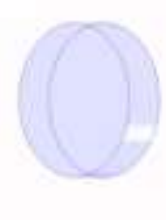

(k)

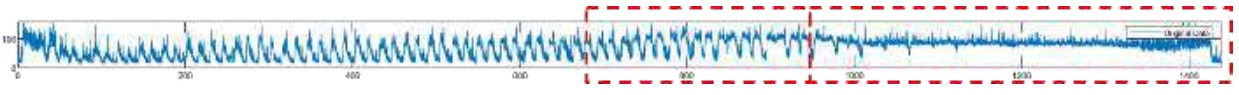

(1)

Fig 4. Steps of the material removal process for a square lens.

The area removed increases as each corner is rotated to the grinding point (Fig. 4[a]-[e]). When corner 2 is rotated to the grinding point for a second time, the part that has already been removed should be deducted. If the length of the lens is $l(\mathrm{~mm})$, depending on the geometry of the lens, the grinding wheel advances $l\left(\frac{1}{\sqrt{2}}-\frac{1}{2}\right)(\mathrm{mm})$ from the contact point and reaches the cylindrical grinding circle. Then, the grinding wheel and the lens remain in contact, and the continuous removal begins (Fig. 4[k]).

Depending on the signal graph in the time domain, the AE signal increases in intensity as the amount of material removed increases, and the periodic peaks gradually turn into plateaus. When the grinding wheel advances to the cylindrical grinding circle, the signal waveform gradually stabilizes (Fig. 4[1]).

\subsection{Signal Analysis}

In the wavelet analysis, the original signal is decomposed into several signals in different time and frequency scales by the scaled and translated mother wavelet. In wavelet multiresolution analysis, the signal is analyzed in both the frequency and time domains. Therefore, the characteristics of the AE signal extracted through wavelet analysis can be used to monitor the grinding process.

This study selected the Daubechies 3 wavelet [27] as the mother wavelet. AE signals were decomposed into one approximate layer and seven detailed layers. The energy coefficient of each detailed layer was calculated to compare the signals. Fig. 5 presents the results of the signal decomposition. 


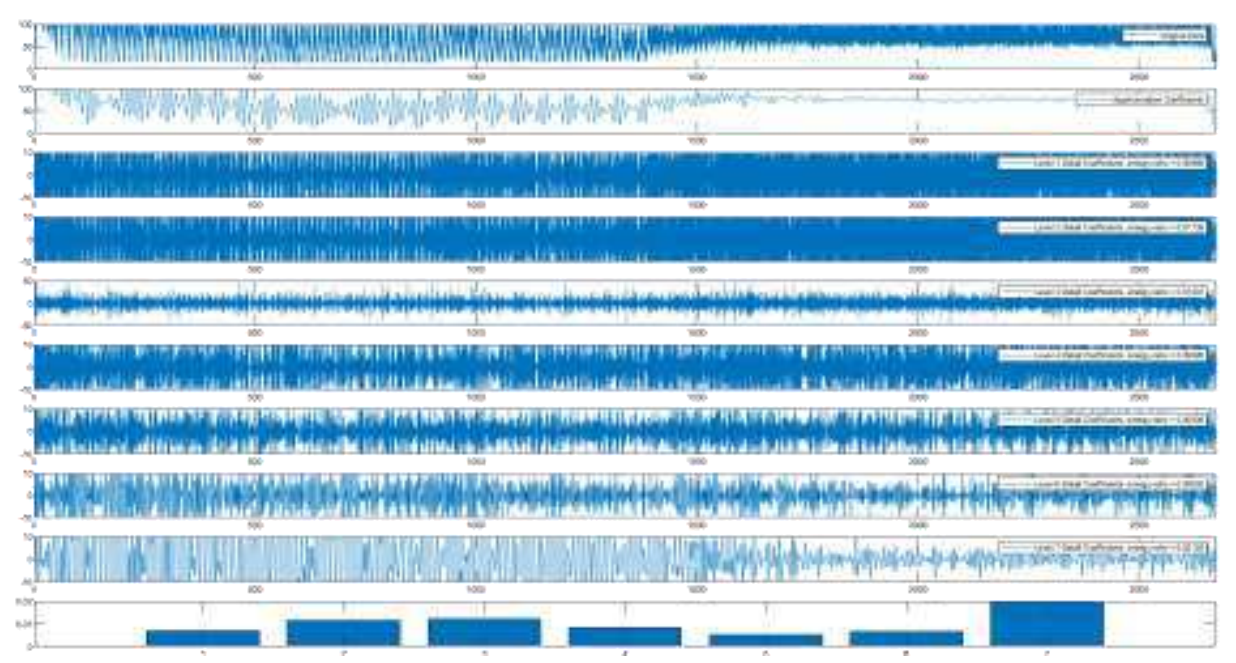

Fig 5. Seven-layer wavelet decomposition

The core of SVMs is the Kernel function. Common Kernel functions include the linear, polynomial, and Gaussian radial basis functions. This study constructed an SVM model on the basis of a linear Kernel function [28]. The quality of the grinding wheel was divided into "worn" and "normal" states on the basis of the abrasive grain distribution on the surface of the grinding wheel.

According to the wavelet analysis, the most significant characteristic coefficients were E3 and E7. According to the theory of wavelet analysis, the lower the detailed layer, the more high-frequency signal characteristics are covered. E7 is associated with low-frequency vibration, which results from machine vibration and ambient noise. E3 is more strongly associated with material removal. The RMS value is a useful reference value for analyzing time-domain features. Therefore, E3 and the RMS value were selected as the classification data set for the SVM.

Fig. 6 presents the data classification of the SVM model. "Normal" and "worn" wheels are indicated by +1 and -1 , respectively. The horizontal axis is the RMS value of the AE signal in the time domain, and the vertical axis is the energy characteristic coefficient extracted through the wavelet analysis. The RMS and energy characteristic coefficient values were normalized to equalize the scales. The left side of the hyperplane is the area representing the "normal" state of the grinding wheel, whereas the right side represents the "worn" state.

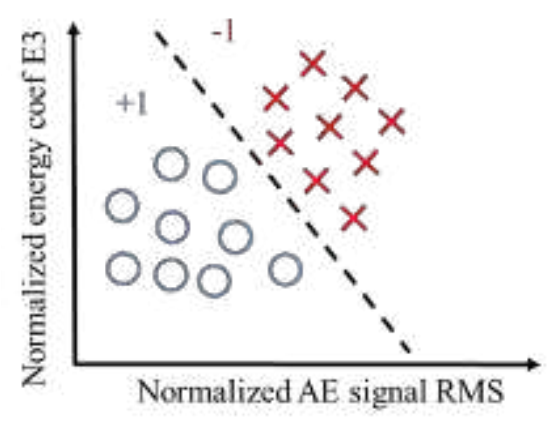

Fig 6. Schematic diagram of the SVM model 


\section{Experiments}

\subsection{Equipment and specifications}

The BE-WF-502N NC (Shonan Optics, Japan) centering machine was used as the experimental platform, and the hydrophone-type $\mathrm{AE}$ sensor was installed to obtain $\mathrm{AE}$ signals during grinding. The sensor was connected to the nozzle of the cutting fluid pipe. An AE signal was generated in the grinding position and transmitted through the medium of the cutting fluid to the AE sensor. After preprocessing steps, such as amplification and filtering, the data were transferred to a computer for analysis. Fig. 7 presents the experimental setup.

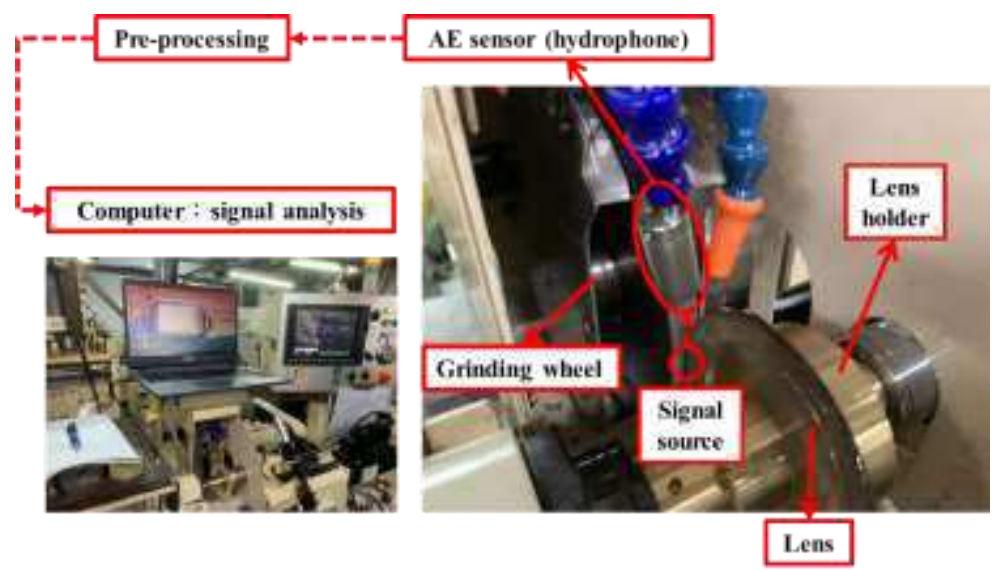

Fig 7. Experimental setup \& schematic

\subsection{Design of the Taguchi Experiment}

The Taguchi method of robust design requires relatively few experiments to determine the influence of process factors on workpiece quality [29-31]. This study used the Taguchi method to design a three-factor, three-level experiment (Table 1). The effects of the holder parameters on the quality of the processed lens were evaluated. In Fig. $8, \theta$ represents the deviation angle of the optical axis, $x$ represents the edge thickness difference of the lens in micrometers, and $d$ represents the holder diameter in millimeters. Factor $\theta$ was considered one of the control factors, but it is difficult to directly measure. Therefore, the ratio of $x$ to $d$, which represents the holder error ratio in Table 1, was used as a control factor instead of $\theta$. Because the effect of holder material on lens quality is substantial, the Young's modulus (MPa) of the holder material was selected as a control factor.

According to Eq. (1), feed rate is a factor affecting the MRR, which directly affects the processing time. Consequently, among the working parameters of the centering machine, the feed rate was used as a control factor. Lenses with different specifications require an appropriate rotation speed for the grinding wheel. If the speed is too high, the lens is easily burned by friction heat. Low speed results in cracks on the surface of the lens due to compressive stress. This study selected a grinding wheel speed of $2400 \mathrm{rpm}$, which is suitable for quartz. 


\begin{tabular}{|c|c|c|c|}
\hline \multicolumn{4}{|c|}{ Table 1. } \\
\hline & Young's modulus (MPa) & Holder error ratio $(\mu \mathrm{m} / \mathrm{mm})$ & Grinding feed rate $(\mathrm{mm} / \mathrm{s})$ \\
\hline 1 & 1 & 1 & 1 \\
\hline 2 & 1 & 2 & 2 \\
\hline 3 & 1 & 3 & 3 \\
\hline 4 & 2 & 1 & 2 \\
\hline 5 & 2 & 2 & 3 \\
\hline 6 & 2 & 3 & 1 \\
\hline 7 & 3 & 1 & 3 \\
\hline 8 & 3 & 2 & 1 \\
\hline 9 & 3 & 3 & 0.005 \\
\hline Level 1 & 105 (Brass ) & 0.01 & 0.015 \\
\hline Level 2 & 200 (Carbon-steel ) & 0.005 & 0.025 \\
\hline Level 3 & 575 (Teflon) & 0.001 & \\
\hline
\end{tabular}

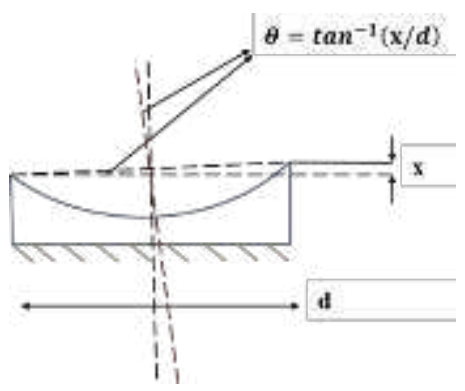

Fig 8. Relationship between the edge thickness difference and optical axis error.

\subsection{Grinding Wheel Wear Experiment}

This study tested an alpha diamond FQT2-459 single-layer electroplated diamond grinding wheel 300 times. The surface quality of the grinding wheel, which was either "normal" or "worn," was observed using the Micro-Vu image measuring instrument. The RMS value of the AE signal and the third-layer energy ratio, E3, obtained through the wavelet analysis were recorded as reference values for labeling and classification. The SVM model was trained on 216 experimental data points to classify the hyperplane into "normal" and "worn" grinding wheel states, and the SVM model was tested using 84 data points to evaluate the learning accuracy.

Fig. 9(a) displays the surface of the grinding wheel before the experiments, whereas Fig. 9(b) shows its surface after the 300 experiments (720,000 rotations). The silver parts in the images are diamond abrasives, the gray part is the metal substrate, and the black parts are the holes where the abrasives fell off. The difference between the images indicates substantial loss of abrasives on the surface of the grinding wheel.

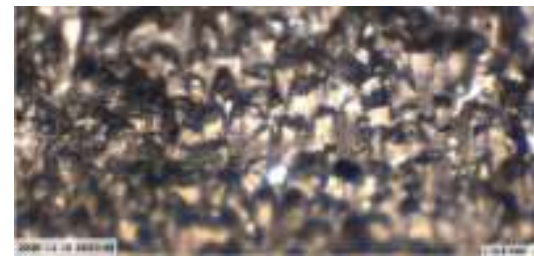

(a)

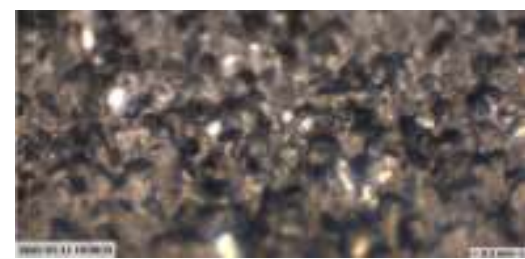

(b)

Fig. 9. Grinding wheel's surface (a) before processing and (b) after 300 experiments. 


\section{Results and Discussion}

\subsection{Material Removal and Shape Reconstruction}

Fig. 10 displays part of the AE signal divided into separate lens rotation cycles. The four peaks in each cycle correspond to the four corners of the square lens; at these points, the MRR was relatively high. The AE signal was positively correlated with the MRR; that is, the cumulative value of the AE signal was relative to the amount of material removed from the lens. The AE signals at each lens angle during each rotation speed were summed. The cumulative value was used to analyze the amount of material removed and the grinding condition at each angle of the lens.

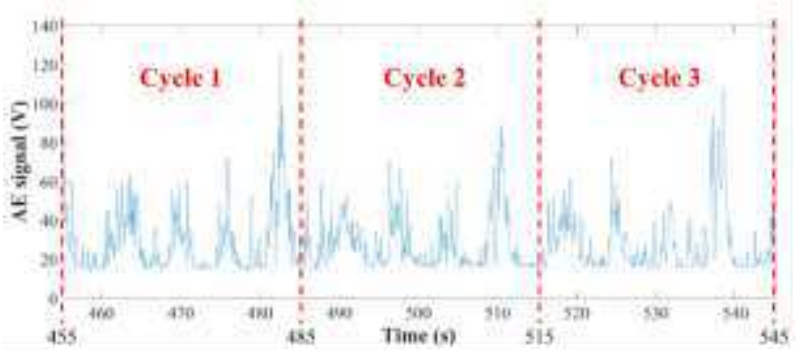

Fig. 10 AE signal of three lens rotations.

The AE signal was transmitted through the cutting fluid concentrated at the cutting point of the grinding wheel. The AE signals consisted of the signals created through material removal and the basic grinding wheel vibration, which had to be subtracted in the analysis. The feed rate of the grinding wheel, flow rate of the cutting fluid, and distance between the grinding wheel and lens affected the basic level of the signal. The basic level of the AE signal differed in every cycle of the lens rotation because the basic amplitude of the $\mathrm{AE}$ signal caused by grinding wheel vibration oscillated rather than remained constant. The basic level was the sum of the minimum value and a correction constant. To obtain the cumulative value along the lens rotation angle, the $\mathrm{AE}$ signal was converted into a polar plot in the angular domain. The signals at the same angle were summed.

Because AE signals are positively correlated with the MRR, the ratio between the cumulative values at different angles should be similar to the ratio of the removal length. In this experiment, the lens width and height before centering were 42.20 and $41.30 \mathrm{~mm}$, respectively. The lens diameter after centering was $37.60 \mathrm{~mm}$. Fig. 11(a)-(c) represent the removal lengths at the corner, upper side, and right side, respectively.

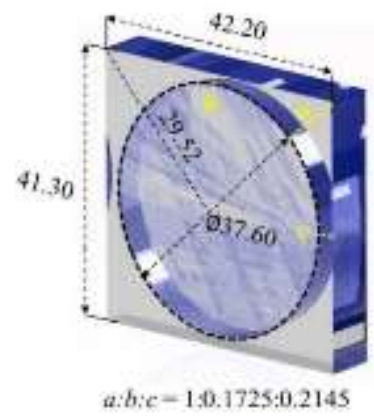

Fig 11. Length ratio of the lens 
The correction constant and the scale from the AE signal to the removal rate were evaluated on the basis of the length ratio a:b:c, which is ideally 1:0.1725:0.2145. Upon comparison of the processing time with the AE signal, a time difference was identified. As feeding of the grinding wheel began, the lens was rotated for an angular shift. Before the correction constant and scale were evaluated, we compensated with an angular shift of 0.2 rad.

The original shape of the square lens before the process was reconstructed, with an evaluated correction constant of $30.2 \mathrm{~V}$ and a scale of 5. Fig. 12(a) presents the original AE signal and the basic level. The length ratio a:b:c was 1:0.1733:0.2155, and the error in the reconstruction was 17.87\%. Fig. 12(b) displays the cumulative AE polar plot, fitted shape, and ideal shape.

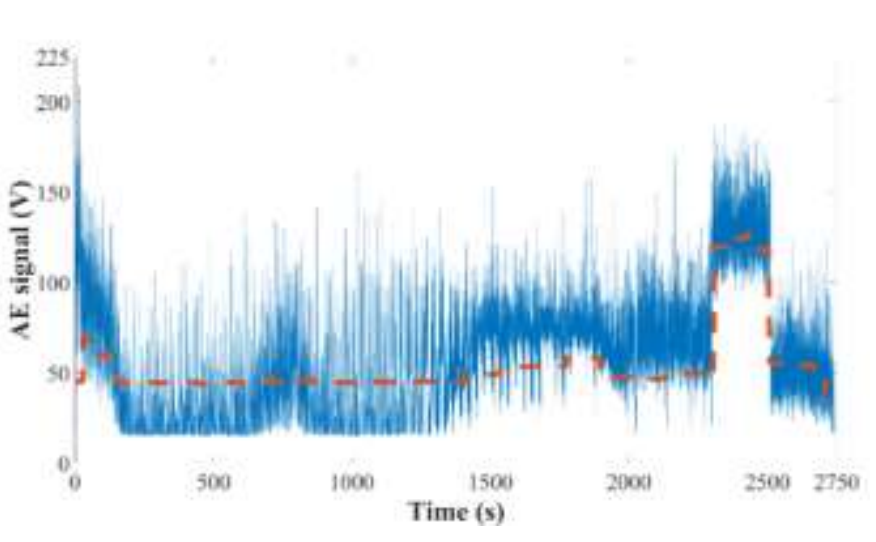

(a)

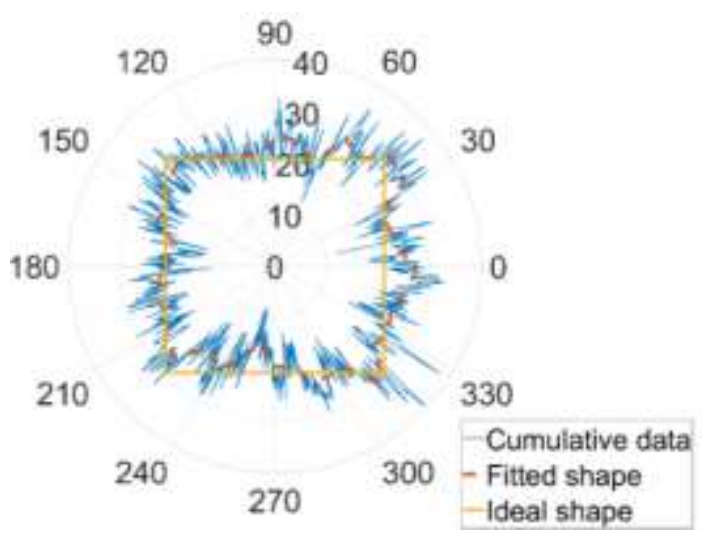

(b)

Fig. 12. (a) AE signal and basic level. (b) Fitted shape of original square lens.

Errors between the ideal shape and fitted shape mainly occurred on the right margin, upper-right margin, and lowerleft corner. On the right and upper-right margins, the amount of rebuilt material was greater than the ideal one. This result indicated that the estimated amount of removed material was greater than the ideal amount. At the lower-left corner, the estimated amount of removed material was smaller than the ideal amount. An edge defect was identified on the centered lens (Fig. 13). The defect consisted of a large amount of removed material, corresponding to angles $0^{\circ}-30^{\circ}$ in Fig. 12(b). The excessive removal at $60^{\circ}$ was offset by insufficient removal at $240^{\circ}$ (Fig. 12[b]). The offset maintained the diameter at $60^{\circ}$, which is close to the average diameter, but caused eccentricity at $60^{\circ}$. The variation in the cumulative data indicated that cracks were generated during the process. According to an inspection of the lens after process, the cracks were shorter than $0.1 \mathrm{~mm}$.

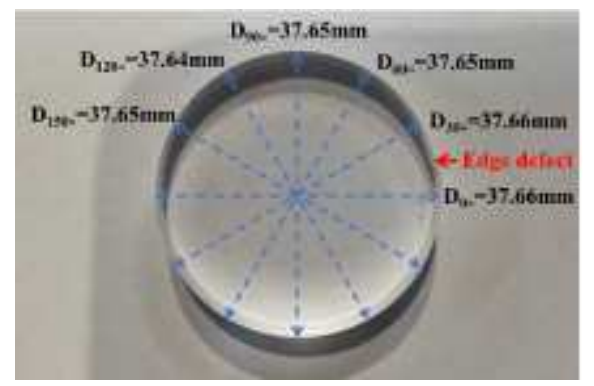

Fig. 13 Biplane quartz lens after the process and the diameter measured at different angles 


\subsection{Wavelet Analysis}

The wavelet energy ratio of each detailed layer $E_{i}$ was calculated using the following equations:

$$
\begin{gathered}
E=E_{I}^{A}+E_{I}^{D} \\
E_{i}=\frac{D_{i}^{2}}{E}, \mathrm{i}=1,2, \ldots \mathrm{I} .
\end{gathered}
$$

where $E$ is the total energy of the wavelet, $I$ is the number of detailed layers, $E_{I}^{A}$ is the approximate layer energy, $E_{I}^{D}$ is the sum of the energies of all detailed layers, and $D_{i}$ is the coefficient value of each detailed layer.

Fig. 14 presents the wavelet energy coefficient distribution obtained from the experiments. B1-B3 refer to the experiments using brass holders, CS1-CS3 refer to the experiments using carbon steel holders, and T1-T3 refer to the experiments using Teflon holders. E7 is the most significant energy ratio. The high E7 values for B1-B3 indicated that the AE signals in the experiments using brass holders contained more low-frequency vibration components, which are considered noise due to mechanical vibration.

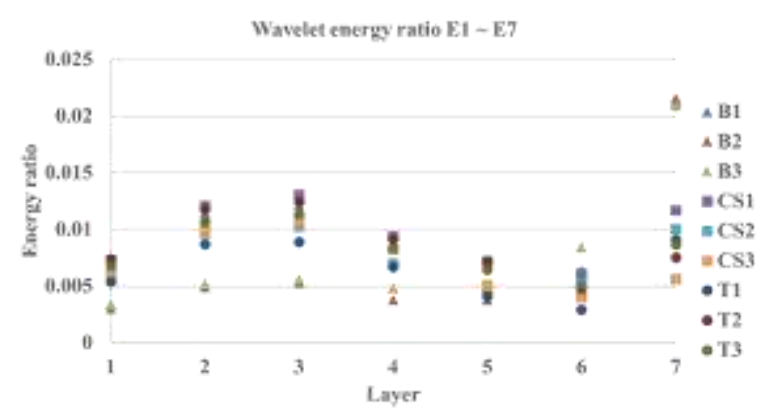

Fig. 14 Wavelet energy coefficient distribution of experiments.

\subsection{Experiment Analysis}

This study designed a Taguchi experiment to analyze the influence of each factor on lens quality. Table 2 presents the corresponding L9 orthogonal table.

\begin{tabular}{|c|c|c|c|c|c|c|c|c|c|c|}
\hline \multicolumn{19}{|c|}{ Table 2. } \\
\hline & \multicolumn{3}{|c|}{ Controlled factors } & \multicolumn{9}{c|}{ Output factors } \\
\hline & $\begin{array}{c}\text { Young's } \\
\text { modulus } \\
(\mathrm{GPa})\end{array}$ & $\begin{array}{c}\text { Holder } \\
\text { error ratio }\end{array}$ & $\begin{array}{c}\text { Feed rate } \\
(\mathrm{mm} / \mathrm{s})\end{array}$ & $\begin{array}{c}\text { Lens } \\
\text { Roundness } \\
(\mathrm{mm})\end{array}$ & $\begin{array}{c}\text { Optical } \\
\text { axis error } \\
(\text { () }\end{array}$ & $\begin{array}{c}\text { AE } \\
\text { RMS } \\
(\mathrm{V})\end{array}$ & $\begin{array}{c}\text { Crack } \\
\text { length } \\
(\mathrm{mm})\end{array}$ & $\begin{array}{c}\text { Scratch } \\
\text { length } \\
(\mathrm{mm})\end{array}$ & $\begin{array}{c}\text { Wavelet } \\
\text { E7 }\end{array}$ & $\begin{array}{c}\text { Ground } \\
\text { surface } \\
\text { roughness } \\
(\mu \mathrm{m})\end{array}$ \\
\hline 1 & 105 & 0.0100 & 0.005 & 0.0065 & 17.219 & 74.2123 & 0.4 & 0.8 & 0.02122 & 0.7032 \\
\hline 2 & 105 & 0.0005 & 0.010 & 0.0110 & 18.727 & 76.3777 & 0.3 & 0.0 & 0.02156 & 0.8320 \\
\hline 3 & 105 & 0.0001 & 0.015 & 0.0215 & 17.588 & 77.1766 & 0.2 & 0.5 & 0.02090 & 0.7617 \\
\hline 4 & 200 & 0.0100 & 0.010 & 0.0050 & 25.918 & 67.0931 & 0.5 & 1.0 & 0.01170 & 0.7177 \\
\hline 5 & 200 & 0.0005 & 0.015 & 0.0035 & 6.780 & 69.1701 & 0.1 & 1.0 & 0.01004 & 0.7422 \\
\hline 6 & 200 & 0.0001 & 0.005 & 0.0010 & 8.628 & 73.6799 & 0.3 & 0.8 & 0.00560 & 0.7437 \\
\hline 7 & 575 & 0.0100 & 0.015 & 0.2175 & 28.060 & 73.3669 & 0.3 & 0.8 & 0.00908 & 0.9867 \\
\hline 8 & 575 & 0.0005 & 0.005 & 0.0040 & 22.811 & 68.8179 & 0.2 & 0.5 & 0.00753 & 0.6672 \\
\hline 9 & 575 & 0.0001 & 0.010 & 0.0055 & 22.436 & 70.1859 & 0.3 & 0.0 & 0.00864 & 0.7737 \\
\hline
\end{tabular}


Table 3 presents the instruments used to measure lens quality.

\begin{tabular}{|c|c|c|}
\hline \multicolumn{3}{|c|}{ Table 3. } \\
\hline Lens quality & Instrument & Precision $(\mu \mathrm{m})$ \\
\hline Optical axis error & Trioptics OptiCentric & 0.1 \\
\hline Crack length/Scratch length & Magnifier & 100 \\
\hline Ground surface roughness & TR200 Surface Roughness Tester & 0.01 \\
\hline
\end{tabular}

In the Taguchi method, the signal-to-noise $(\mathrm{S} / \mathrm{N})$ ratio is a useful indicator when attempting to improve the robustness of a process and minimize the effect of noise on the output. The parameters can be adjusted to stabilize the process if the $\mathrm{S} / \mathrm{N}$ ratio is relatively large. Fig. 15 presents the $\mathrm{S} / \mathrm{N}$ ratio of each control factor, where $E$ is the Young's modulus of the holder material, $P V$ is the holder error ratio, and $F R$ is the grinding wheel feed rate. The outputs of the experiments were the-smaller-the-better because they represented lens defects or mechanic vibration signals.

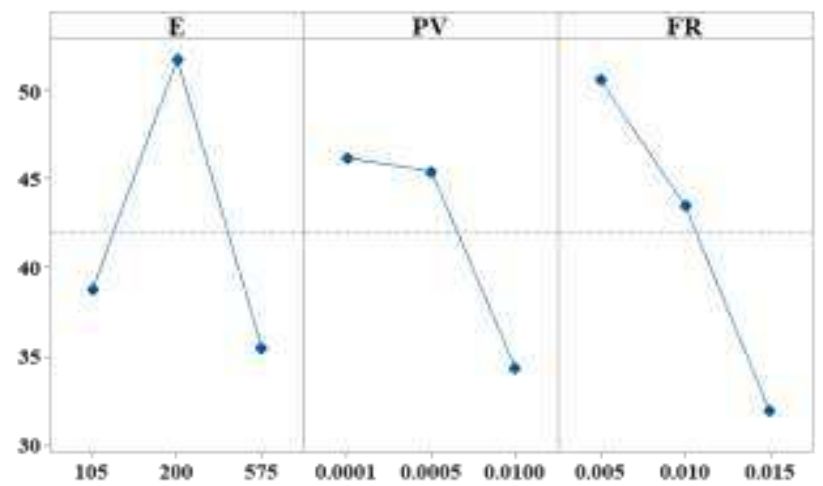

(a)

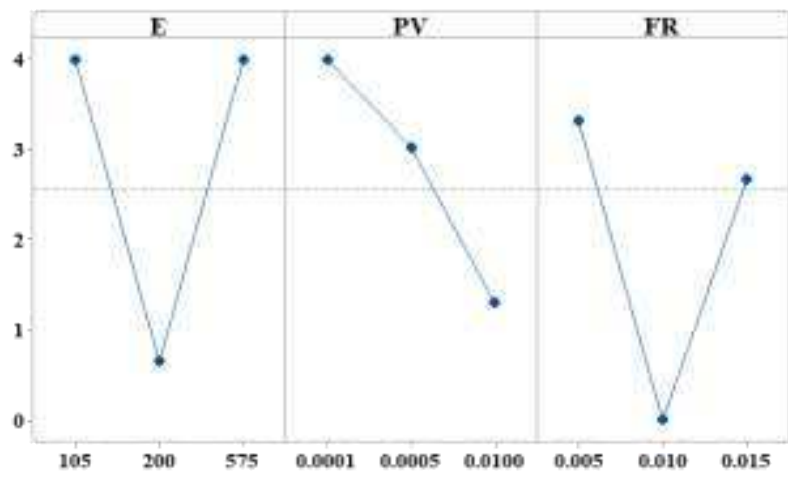

(c)

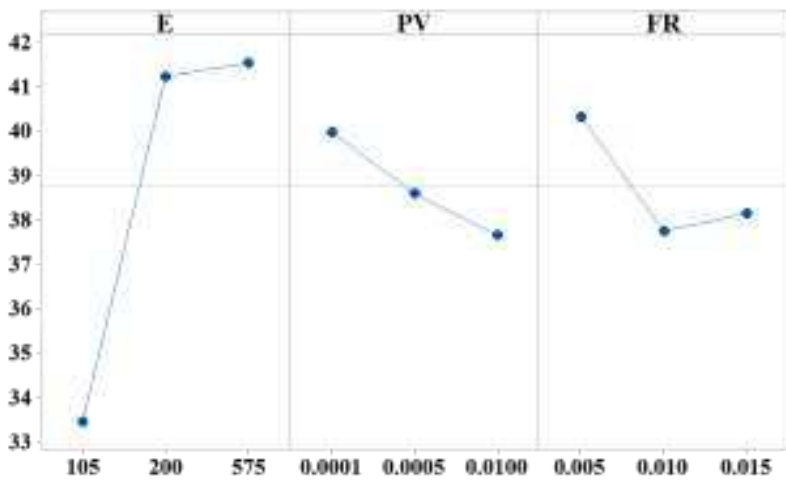

(b)

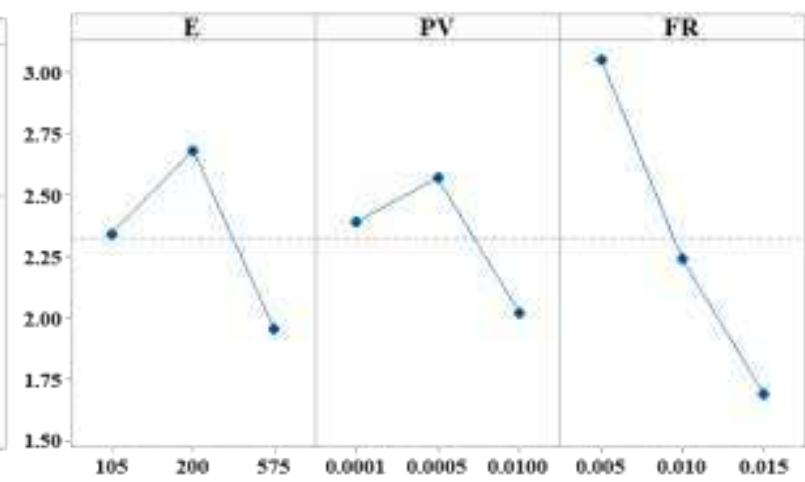

(d) 


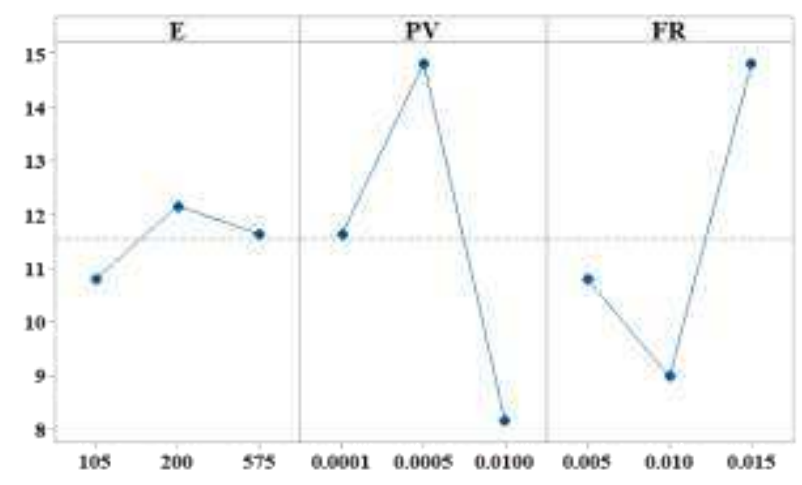

(e)

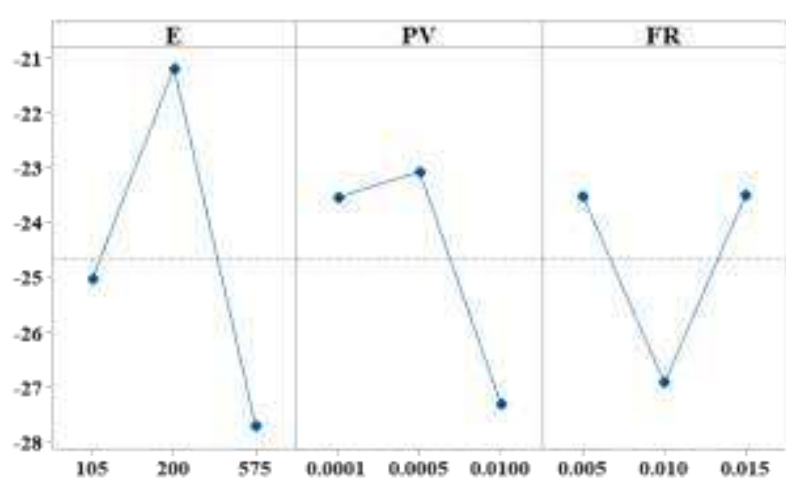

(f)

Fig. 15. S/N ratio of each output (the-smaller-the-better). (a) Circularity error. (b) Wavelet energy ratio E7. (c) Scratch length. (d) Ground surface roughness. (e) Crack length. (f) Optical axis error.

The S/N ratio indicated how the holder material, edge error ratio, and wheel feed rate affected the lens process (Fig. 15). The brass holders were easily deformed because of the low Young's modulus of brass. The deformed holders decentered the lens and caused external vibration, which was a low-frequency component of the AE signal. Consequently, E7 had the largest wavelet energy coefficient, and the lens roundness was poor. The carbon steel holders could hold the lens stably and decreased the grinding vibration because of carbon steel's high stiffness and friction coefficient. The optical axis error, edge crack length, and grinding surface roughness were superior to those generated in the tests using brass holders. However, the polished surface was damaged by the holders because of carbon steel's high stiffness and friction coefficient. The Teflon holders had the largest Young's modulus, and the friction coefficient of this material was low. The Teflon holders barely scratched the lens or derailed the working process. However, because Teflon's low surface strength caused the holder to be damaged easily, the holder's lifetime was short. In addition, the extremely low friction of the material meant that the normal force of the grinding wheel could not be resisted by the holder. If the feed rate had been too high, the lens would have been pushed away from the working axis.

A holder with an edge error indicates that the contact area between the holder and the lens' polished surface is uneven. This condition increases the compressive stress between the holder and lens and thus increases the length of scratches on the surface of the lens. However, the compressive stress on the surface of the lens represents the holding force of the holder, and such force stabilizes the grinding process. Therefore, the grinding quality is more stable with a holder edge error of 0.0005 than with a holder edge error of 0.0001 . However, an edge error of 0.01 is sufficiently large to strongly affect decentering of the edge surface when the lens is rotated. Therefore, the optimal holder error ratio is between 0.0001 and 0.0005 .

In the case of a fixed grinding wheel speed, the lower the feed rate, the less material removed per revolution and the smoother the surface of the ground lens. Because the surface of a sintered diamond grinding wheel is uneven, the vibration it causes is large. However, the larger the feed rate, which is proportional to the depth of cut, the more strongly the vibration signal can be suppressed. Therefore, when the feed rate is $0.015 \mathrm{~mm} / \mathrm{s}$, the shortest cracks are obtained. 


\subsection{SVM Classification Model}

Under identical parameters, the amplitude of the AE signal increased with the processing time. The grinding mechanism gradually may have changed from grinding to friction and normal stress because of wear of the grinding abrasives, which destabilizes the process (Fig. 16).

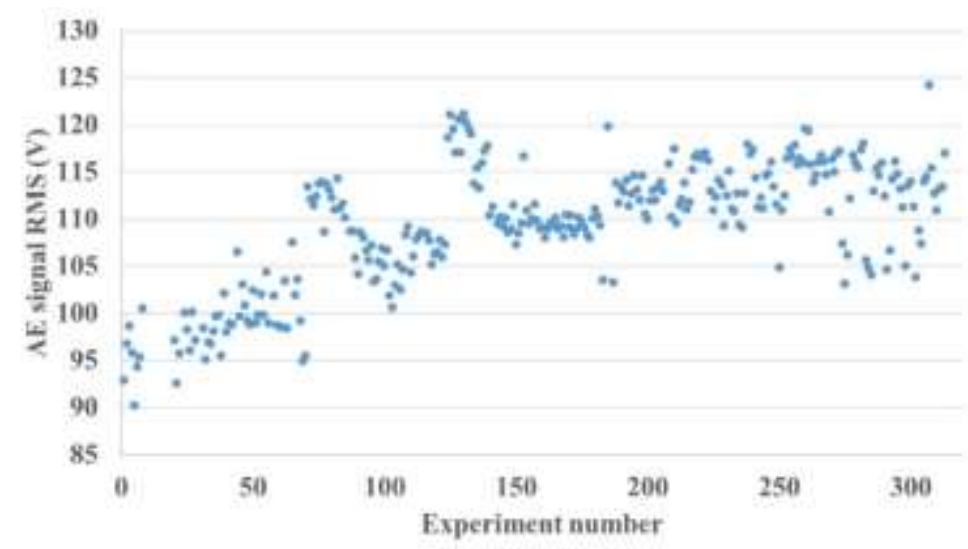

Fig. 16 Distribution of AE signal's RMS

The RMS is a useful indicator for evaluating the wear on a grinding wheel's abrasives. However, a start-up centering machine works with a relatively high RMS value. The process was halted at experiment numbers 50 and 100 and restarted with an RMS value approximately $20 \mathrm{~V}$ higher than the previous value (Fig. 16). The RMS alone is not sufficient to accurately evaluate the degree of wear. Another indicator should be used to improve the accuracy of the evaluation. As discussed in Section 2.2, in wavelet analysis, E3 is associated with material removal. Therefore, the E3 coefficient and RMS value of the AE signal were employed to construct the classification data set for the SVM.

The experimental data were separated into two groups. The first 150 experimental data points were marked as indicating a "normal" wheel surface $(+1)$, whereas the last 150 experiment data points were marked as indicating a "worn" surface (-1). The SVM model was trained and tested using the data, with the outliers being filtered out beforehand. The classification accuracy was as follows:

Accuracy $=$ (correctly predicted data / total testing data $) \times 100 \%$.

Fig. 17 presents the results of the linear classification. The SVM model classified wheels into "normal" and "worn" groups with an accuracy of $89 \%$ when outliers were included and $95 \%$ when they were excluded.

As the grinding wheel became worn, both the RMS and wavelet feature E3 of the AE signal increased. The data points were distributed toward the upper right of the diagram. Consequently, the hyperplane that separated the data points into the "worn" and "normal" states was a line with negative slope. The margins on both sides of the hyperplane were a certain distance from the hyperplane. The data points between the margins indicated that a normal wheel was going to become worn or that a worn wheel was not completely worn. 


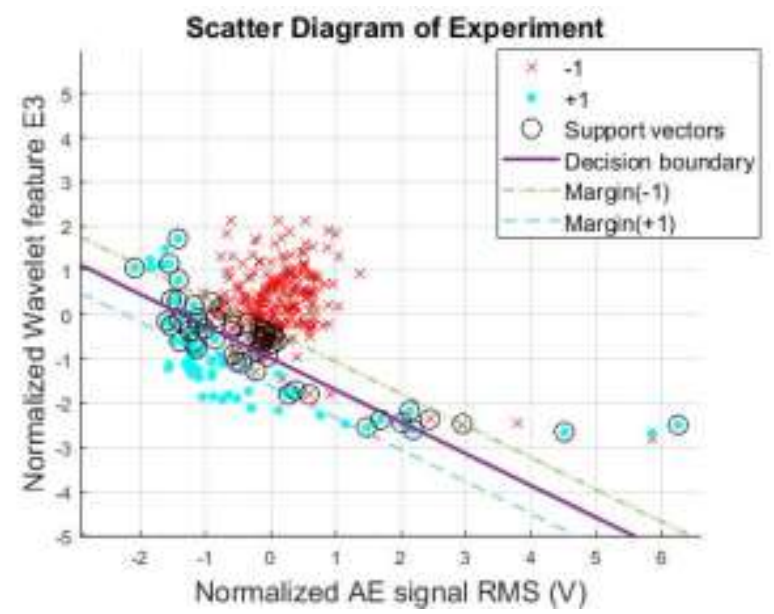

(a)

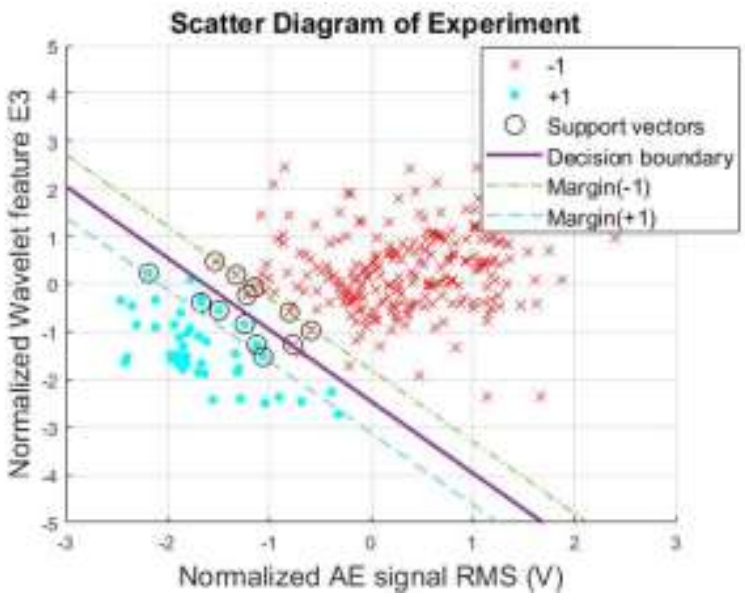

(b)

Fig. 17. Classification results: (a) when including outliers, accuracy $=95.6989 \%$ (89/93); (b) when excluding outliers, accuracy $=98.8095 \%(83 / 84)$.

With the classification model, the hyperplane could be used to evaluate the surface condition of a grinding wheel and make appropriate decisions. The condition of the grinding wheel can be determined, and the appropriate operational decision can be made by examining the distribution of data collected in real time relative to the hyperplane. Worn grinding wheels can be repaired or replaced before grinding is resumed to ensure the quality of the processed lens.

According to the results of the experiment, a square quartz biplane lens could be processed with optimal centering parameters. The MRR was monitored, and the lens shape was reconstructed through data processing. The quality of the surface of the grinding wheel was controlled by applying the SVM model. With this analysis method, the centering process and additional inspection procedures can be optimized. According to the results of the experiment, the quality of the lens can be improved to an edge surface roughness of $<2 \mu \mathrm{m}$, a lens circularity error of $<0.01 \mathrm{~mm}$, a crack length of $<$ E 0.1 , and an optical axis error of $<150 \mu \mathrm{rad}$.

\section{Conclusion}

This study proposed a method for analyzing the optical quartz lens centering process. The MRR was monitored using a hydrophone AE sensor. The shape of the original square lens was reconstructed using accumulated AE signals obtained in the angular domain of the lens. With a rebuilding error of $17.87 \%$, cracks, eccentricity, and edge defects were verified through comparison with the processed lens.

An L9 Taguchi experiment was designed to analyze the effects of the lens holder's parameters and the grinding wheel feed rate on lens quality. Wavelet analysis was performed to obtain the energy coefficient for different frequency domains. By combining a Taguchi experiment and wavelet analysis, the process parameters were optimized. An SVM classification model was constructed and trained to monitor grinding wheel quality; the model had accuracy of $98.8 \%$.

By using this analysis method, the quality of the workpieces can be controlled to an edge surface roughness of $<2$ $\mu \mathrm{m}$, a lens circularity error of $<0.01 \mathrm{~mm}$, a crack length of $<\mathrm{E} 0.1$, and an optical axis error of $<150 \mu \mathrm{rad}$. 


\section{Declarations}

\section{a. Funding}

The authors are grateful for the support of the Research Project of the Ministry of Science and Technology, Taiwan (MOST 108-2218-E-007-059-MY2 and MOST 109-2622-E-007-028).

\section{b. Conflicts of interest/Competing interests}

The authors declare no competing interests.

c. Availability of data and material

Not applicable.

\section{d. Code availability}

Not applicable.

e. Ethics approval

Not applicable.

\section{f. Consent to participate}

All authors were fully involved in the study and preparation of the manuscript; each of the authors has read and concurs with the content in the final manuscript.

\section{g. Consent for publication}

All authors consent to publish the content in the final manuscript.

\section{h. Authors' contributions}

Chun-Wei Liu: Conceptualization, Supervision

Shaiu-Cheng Shiu: Quartz lens shape reconstruction, Reviewing and Editing

Kai-Hung Yu: SVM classification, Experiment Design, Writing

\section{References}

[1] C. Jiang, H. Li, Y. Mai, D. Guo (2013) Material Removal Monitoring in Precision Cylindrical Plunge Grinding Using Acoustic Emission Signal, ARCHIVE Proceedings of the Institution of Mechanical Engineers Part C Journal of Mechanical Engineering Science 228(4):715-722.

[2] W. Hundt, D. Leuenberger, F. Rehsteiner, P. Gygax (1994) An Approach to Monitoring of the Grinding Process Using Acoustic Emission (AE) Technique, CIRP Annals 43(1):295-298.

[3] M. Ahrens, R. Fischer, M. Dagen, B. Denkena, T. Ortmaier (2013) Abrasion Monitoring and Automatic Chatter Detection in Cylindrical Plunge Grinding. Procedia CIRP 8:374-378.

[4] N. Ding, J.S. Duan, C. Liu, S. Jiang, S.F Cui, Experimental Study on the Relationship Between Acoustic Emission Signal and Grinding Wheel Wear, IEEE Advanced Information Technology, Electronic and Automation Control Conference (IAEAC), 20-22 Dec. 2019, Chengdu, China.

[5] D. Dornfeld, H. Cai (1984) An Investigation of Grinding and Wheel Loading Using Acoustic Emission, Journal of Engineering for Industry 106(1):28-33.

[6] W. L. Weingaertner, A. Boaron (2012) A method to determine the grinding wheel's topography based on acoustic 
emission, International Journal of Abrasive Technology 5(1):17-32.

[7] C.S. Liu, Y.J. Ou. (2020) Grinding Wheel Loading Evaluation by Using Acoustic Emission Signals and Digital Image Processing. Sensors 20(15):4092.

[8] J. Badger, S. Murphy, G.E. O'Donnell (2018) Acoustic emission in dressing of grinding wheels: AE intensity, dressing energy, and quantification of dressing sharpness and increase in diamond wear-flat size. International Journal of Machine Tools and Manufacture 125:11-19.

[9] D.F.G. Moia, I.H. Thomazella, P.R. Aguiar, E.C. Bianchi, C.H.R. Martins \& M. Marchi (2014) Tool condition monitoring of aluminum oxide grinding wheel in dressing operation using acoustic emission and neural networks. The Brazilian Society of Mechanical Sciences and Engineering 37:627-640.

[10] D.E. Lee, I. Hwang, C.M.O. Valente, J.F.G. Oliveira, D.A. Dornfeld (2003) Precision manufacturing process monitoring with acoustic emission, International Journal of Machine Tools \& Manufacture 46:176-188.

[11] E.S. Lee, J.W. Cha, S.H. Kim (2013) Evaluation of the wafer polishing pad capacity and lifetime in the machining of reliable elevations, International Journal of Machine Tools \& Manufacture 66:82-94.

[12] W.N. Lopes et al. (2017) Digital signal processing of acoustic emission signals using power spectral density and counts statistic applied to single-point dressing operation, IET Science, Measurement \& Technology 11(5).

[13] C.M. Chen, R. Kovacevic, D. Jandgric (2003) Wavelet transform analysis of acoustic emission in monitoring friction stir welding of 6061 aluminum, International Journal of Machine Tools and Manufacture 43(13):1383-1390.

[14] H. Xin, L. Cheng, R. Diender, M. Veljkovic, Fracture acoustic emission signals identification of stay cables in bridge engineering application using deep transfer learning and wavelet analysis, Advances in Bridge Engineering 1 (2020).

[15] K. He, Z. Xia, Y. Si, Q. Lu, Y. Peng (2020) Noise Reduction of Welding Crack AE Signal Based on EMD and Wavelet Packet, Sensors 20(3):761.

[16] C.U. Grosse, F. Finck, J.H. Kurz, H.W. Reinhardt (2004) Improvements of AE technique using wavelet algorithms, coherence functions and automatic data analysis, Construction and Building Materials 18(3):203-213.

[17] V. Pandiyan, T. Tjahjowidodo (2017) In-process endpoint detection of weld seam removal in robotic abrasive belt grinding process, The International Journal of Advanced Manufacturing Technology 93:1699-1714.

[18] S.Y. Cho, S. Asfour, A. Onar, N. Kaundinya (2005) Tool breakage detection using support vector machine learning in a milling process, International Journal of Machine Tools \& Manufacture 45:241-249.

[19] E. Sauter, E. Sarikaya, M. Winter, K. Wegener (2021) In-process detection of grinding burn usingmachine learning, The International Journal of Advanced Manufacturing Technology 115:2281-2297.

[20] H. B. Hübner, M. A.V. Duarte1, R. B. da Silva (2020) Automatic grinding burn recognition based on time-frequency analysis and convolutional neural networks, The International Journal of Advanced Manufacturing Technology 110:1833-1849.

[21] W. Guo, B. Li, S. Shen, Q. Zhou (2019) An intelligent grinding burn detection system based on two-stage feature selection and stacked sparse autoencoder, The International Journal of Advanced Manufacturing Technology 103:28372847.

[22] J. Guo (2014) Surface roughness prediction by combining static and dynamic features in cylindrical traverse grinding, The International Journal of Advanced Manufacturing Technology 75:1245-1252. 
[23] Q. Gu, Z. Deng, L. Lv, T. Liu, H. Teng, D. Wang, J. Yuan (2021) Prediction research for surface topography of internal grinding based on mechanism and data model, The International Journal of Advanced Manufacturing Technology 113:821-836.

[24] Z. Chang, Q. Jia, X. Yuan, Y. Chen (2017) Optimization of the grinding process to improve the surface integrity of bearing raceways, The International Journal of Advanced Manufacturing Technology 91:4243-4252.

[25] X. Zhang, C. Krewet, B. Kuhlenkotter (2006) Automatic classification of defects on the product surface in grinding and polishing, International Journal of Machine Tools \& Manufacture 46:59-69.

[26] I.D. Marinescu, W.B. Rowe, B. Dimitrov, I. Inasaki. (2004) TRIBOLOGY OF ABRASIVE MACHINING PROCESSES. Norwich, NY: William Andrew, Inc.

[27] T. Loutas, G. Sotiriadis, V. Kostopoulos, On the application of wavelet transform of AE signals from composite materials, European Working Group on Acoustic Emission 2004, Berlin, Germany.

[28] Y. Yu, L. Zhou (2012) Acoustic Emission Signal Classification based on Support Vector Machine, TELKOMNIKA Indonesian Journal of Electrical Engineering 10(5):1027-1032.

[29] H. Hernadewita, I. Rochmad, H. Hendra, H. Hermiyetti, E.N.S. Yuliani (2019) An Analysis of Implementation of Taguchi Method to Improve Production of Pulp on Hydrapulper Milling, International Journal of Production Management and Engineering 7(2):125-131.

[30] S. PERIYASAMY (2014) Optimization of Surface Grinding Process Parameters by Taguchi Method and Response Surface Methodology, International Journal of Engineering Research 3(5):1721-1727.

[31] N.C. Deresse, V. Deshpande, I.W.R. Taifa (2020) Experimental investigation of the effects of process parameters on material removal rate using Taguchi method in external cylindrical grinding operation, Engineering Science and Technology, an International Journal 23(2):405-420. 